\title{
ANOMALOUS DIELECTRIC BEHAVIOUR OF La(III) SUBSTITUTED LEAD TITANATE CERAMICS
}

\author{
K. Keizer, G. J. LANSINK and A. J. BuRgGraAF \\ Twente University of Technology, Department of Chemical Engineering, Laboratory of Inorganic Chemistry and \\ Materials Science, P.O. Box 217 Enschede, The Netherlands
}

(Received 24 February 1977; accepted in revised form 13 May 1977)

\begin{abstract}
In $\mathrm{La}(\mathrm{III})$ substituted $\mathrm{PbTiO}_{3}$ ceramics the behaviour of the reciprocal dielectric constant in the paraelectric phase can be described by the relation $1 / \epsilon \sim\left(T-T_{\epsilon}\right)^{\gamma}$ with the exponent $1 \leqslant \gamma<2$ depending on the composition. This $\gamma$-value is an intermediate of values for Curie-Weiss behaviour $(\gamma=1)$ and of values connected with diff use phase transitions $(\gamma=2)$ and increases with increasing La(III) concentration.

An excellent agreement between experimental and calculated permittivity curves in the paraelectric phase can be achieved using an alternative equation consisting of a linear and a quadratic term with coefficients varying systematically with the composition. The last equation can be derived and interpreted by means of a physical model assuming the existence of heterophase fluctuations.
\end{abstract}

\section{INTRODUCTION}

In ferroelectric materials the relative dielectric constant $\epsilon^{\prime}$ rises sharply with increasing temperature to a high peak value at the phase transition temperature $T_{c}$. Above $T_{c}$ the reciprocal dielectric constant as a function of temperature can be frequently represented by a CurieWeiss type equation [1-3].

$$
1 / \epsilon^{\prime}=\left(T-T_{0}\right) / C_{w}
$$

where $C_{w}$ is the Curie-Weiss constant and $T_{0}$ is the extrapolated Curie temperature.

In perovskite $\left(\mathrm{ABO}_{3}\right)$-type materials deviations from Curie-Weiss behaviour are reported [4-8], especially in compounds with a complex occupation of the $A$ and/or $B$-site in the crystal lattice.

Wolters and Burggraaf [4], Fritsberg[6], Smolensky[7] and Kirillov and Isupov [8] have developed models to describe the diffuse character of the ferroelectricparaelectric phase transition (FPT) for this type of materials.

The basic phenomenon which determines the occurrence of diffuse phase transitions is the presence of structural and/or compositional fluctuations in a considerable temperature region around $T_{c^{*}}$ One way of describing this phenomenon quantitatively is by means of a distribution of small volume elements each characterized by a particular value of a relevant parameter of state, e.g. the Curie temperature.

With a Gaussian distribution of Curie temperatures and a variance $C$ the following equation results [4]

$$
1 / \epsilon^{\prime}=1 / \epsilon_{\max }^{\prime}\left(1+\frac{\left(T-T_{\max }\right)^{2}}{2 C^{2}}\right)
$$

where $\epsilon_{\max }^{\prime}$ is the maximum value of $\epsilon^{\prime}$ at the temperature $T_{\max } . C$ is a measure for the degree of diffuseness of the FPT.

In the $\mathrm{PbO}-\mathrm{TiO}_{2}-\mathrm{La}_{2} \mathrm{O}_{3}$ system a perovskite phase exists with the chemical formula $\mathrm{Pb}_{1-\alpha x} \mathrm{La}_{x} \mathrm{TiO}_{3+x(1.5-\alpha)}$ (PLT) [9-12]. The permittivity as a function of temperature in the paraelectric phase (PE) of this system at $\mathrm{La}$ (III) concentrations where $x>0.07$ cannot be described by the eqn (1) or (2). This phenomenon is also reported for niobate systems [5].

This paper deals mainly with a description and an interpretation of this anomalous behaviour.

\section{EXPERIMENTAL.}

Because the physical properties in these types of materials are very sensitive to variations in microstructure (porosity, grain size homogeneity) extreme care must be taken to prepare and characterize the samples. In Refs. $[4,9,10,11]$ an extensive description of the preparation and characterization of the samples has been given and in this paper a summary of a few topics of the used methods is given.

PLT samples for this investigation were prepared by means of the mixed oxide method using a controlled $\mathrm{PbO}$ atmosphere $[9,10]$. A good homogeneity in monophasic materials was achieved and this was apparent from electron microscope examination and from the line width of some characteristic X-ray reflections with respect to a silicon standard[10]. This latter method yields values for the line width which are always smaller than $0.15^{\circ}$ in $\theta$ (in most cases $0.12^{\circ}$ ) compared with values of $0.10^{\circ}-0.12^{\circ}$ for the silicon standard at $\theta<35^{\circ}$.

Special attention was paid to the determination of the $\mathrm{PbO}$ content of the material ( $\alpha$-value) using a very sensitive method of chemical analysis which can discriminate between $\mathrm{PbO}$ at grain boundaries and $\mathrm{PbO}$ incorporated in the structure $[9,11]$. The samples contain less than 0.2 wt.\% "free PbO" which is equal to the inaccuracy of the used method[11]. Possible gradients of $\mathrm{PbO}$ over the sample thickness were minimized by taking thin $(2 \mathrm{~mm})$ sintered samples in such a way that the sample thickness does not play a role (sample surface layer is removed). 
Average grain size were measured by means of scanning electron microscope micrographs using the Mendelsohn method[12].

The capacity and conductivity of the samples were measured by means of Wayne-Kerr Universal Bridges types B221 and B601 at frequencies between $0.5 \mathrm{kHz}$ and $1 \mathrm{MHz}$. Heating and cooling rates varied between $0.5 \mathrm{deg} . / \mathrm{min}$ (at $T \approx T_{c}$ ) and $2.0 \mathrm{deg} . / \mathrm{min}$ (at $T \gg T_{C}$ ).

\section{RESULTS}

In Table 1 the analyzed values of the compositional parameters $x$ and $\alpha$ (in $\mathrm{Pb}_{1-\alpha x} \mathrm{La}_{x} \mathrm{TiO}_{3+x(1.5-\alpha}$ ) are collected together with average grain size diameters $\bar{g}$, unit cell parameters (length of unit cell axis $a$ and tetragonal distortion $c(a)$ at room temperature and dielectric parameters $T_{\max }$ and $\epsilon_{\max }^{\prime}$ characteristic for the behaviour of the FPT. The tetragonal distortion $c / a$ decreases with increasing $x$ and decreasing $\alpha$ and reaches a value of 1.0000 at values of $x \approx 0.27$ depending on the exact $\alpha$ value. There is a continuous decrease of $T_{\max }$ with $x$ of about $20^{\circ} \mathrm{C} / \mathrm{at} \%$ $\mathrm{La}$ (III) and an increase of $T_{\max }$ with $\alpha$ of about $2^{\circ} \mathrm{C} / \mathrm{mole} \%$ $\mathrm{PbO}$ at a constant $x$-value[13].

Table 1. Composition, grain size, lattice dimensions and dielectric properties of various $\mathrm{La}$ (III) substituted $\mathrm{PbTiO}_{3}$ samples

\begin{tabular}{cccccccc}
\hline $\begin{array}{c}\text { Sample } \\
\text { No. }\end{array}$ & $x$ & $\alpha$ & $\left.\begin{array}{c}a \\
(\AA)\end{array}\right)$ & $\begin{array}{c}c l a \\
(-)\end{array}$ & $\begin{array}{c}\bar{g} \\
(\mu \mathrm{m})\end{array}$ & $\begin{array}{c}T_{\max } \\
\left({ }^{\circ} \mathrm{C}\right)\end{array}$ & $\begin{array}{c}\epsilon_{\max } \times 10^{-3} \\
(-)\end{array}$ \\
\hline 1 & 0.040 & 1.30 & 3.9066 & 1.0478 & 1.3 & 407 & 7.8 \\
2 & 0.069 & 1.23 & 3.9112 & 1.0383 & 3.1 & 357 & 13.3 \\
3 & 0.120 & 1.45 & 3.9141 & 1.0263 & 4.7 & 257 & 14.6 \\
4 & 0.120 & 0.83 & 3.9209 & 1.0275 & 4.8 & 273 & 12.7 \\
5 & 0.180 & 1.34 & 3.9184 & 1.0149 & 9.4 & 151 & 16.8 \\
6 & 0.180 & 0.87 & 3.9289 & 1.0166 & 6.4 & 168 & 16.6 \\
7 & 0.230 & 1.05 & 3.9274 & 1.0074 & 10 & 53 & 14.2 \\
8 & 0.270 & 1.48 & 3.9196 & 1.0000 & 8 & -45 & 15.7 \\
9 & 0.270 & 1.18 & 3.9290 & 1.0000 & 15 & -36 & 11.2 \\
10 & 0.301 & 1.41 & 3.9177 & 1.0000 & 10 & -116 & 7.8 \\
11 & 0.301 & 1.20 & 3.9303 & 1.0000 & 14 & -105 & 6.8 \\
\hline
\end{tabular}

For $x \leqslant 0.23$ the permittivity curves are not "broadened" at any $\alpha$-value as is shown for $x=0.18$ in Fig. 1 , but for $x \geqslant 0.27$ the permittivity curves become more "broadened" with increasing $\mathrm{PbO}$ content (decreasing $\alpha$ ) and increasing $x$ value, as is shown in Fig. 2 and Fig. 3 for $x=0.27$ and $x=0.30$ respectively.

Since the permittivity curves show no or only a small frequency dependency, most of the measurements were performed at $10 \mathrm{kHz}$ with a small number of other measurements at $250 \mathrm{kHz}$.

$$
1 / \epsilon^{\prime}=1 / \epsilon_{\max }^{\prime}+C^{\prime}\left(T-T_{\max }\right) \gamma
$$

where $\gamma$ and $C^{\prime}$ are constants and the values of these constants depend on the composition. Equation (3) is valid over a temperature region of at least $60^{\circ}-100^{\circ} \mathrm{C}$ above $T_{\max }$. The value of $\gamma$ increases from 1.0 at $x=$ 0.04 and $x=0.07$ to 1.70 at $x=0.30$ as is shown in Fig. 4. So a change from a Curie-Weiss behaviour to an intermediate of Curie-Weiss (eqn 1) and quadratic behaviour (eqn 2) can be observed.

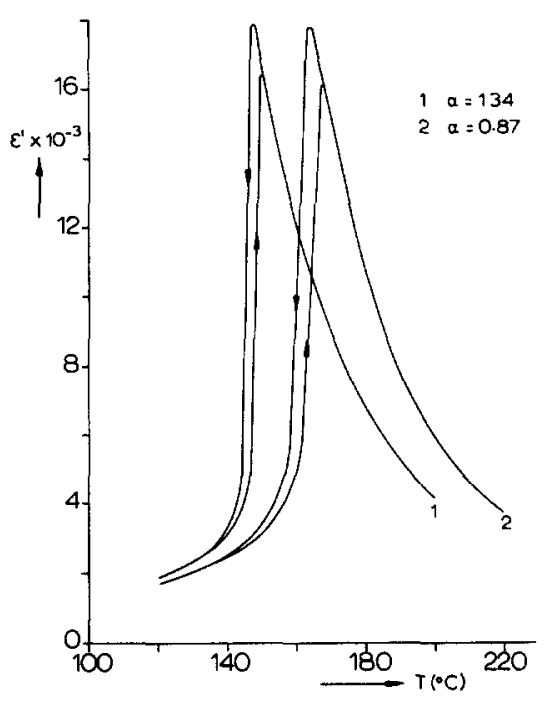

Fig. 1. The effect of the value of $\alpha$ on the $\epsilon^{\prime}$ vs temperature curves near the FPT for PLT with $x=0.18$.

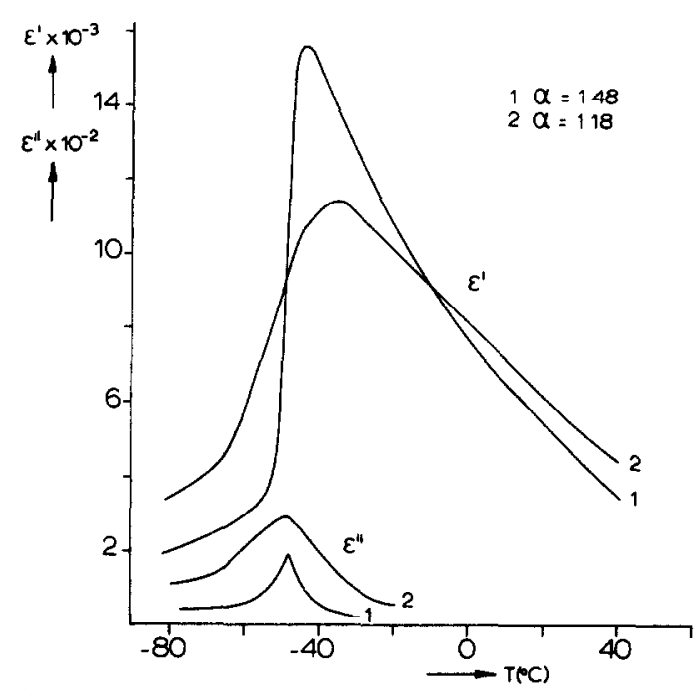

Fig. 2. The effect of the value of $\alpha$ on the $\epsilon^{\prime}$ vs temperature curves near the FPT for PLT with $x=0.27$ ( $\epsilon$ " is dielectric loss).

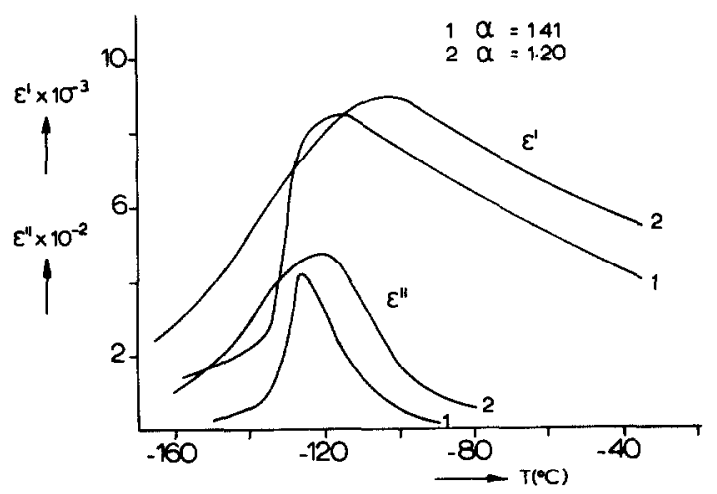

Fig. 3. The effect of the value of $\alpha$ on the $\epsilon^{\prime}$ vs temperature curves near the FPT for PLT with $x=0.30$ ( $\epsilon^{\prime \prime}$ is dielectric loss).

The change in $\gamma$ is not caused by grain size effects as appears from studies by Keizer and Burggraaf $[10,14,15]$. In these studies it was shown that the effect of the grain size on the FPT becomes im- 


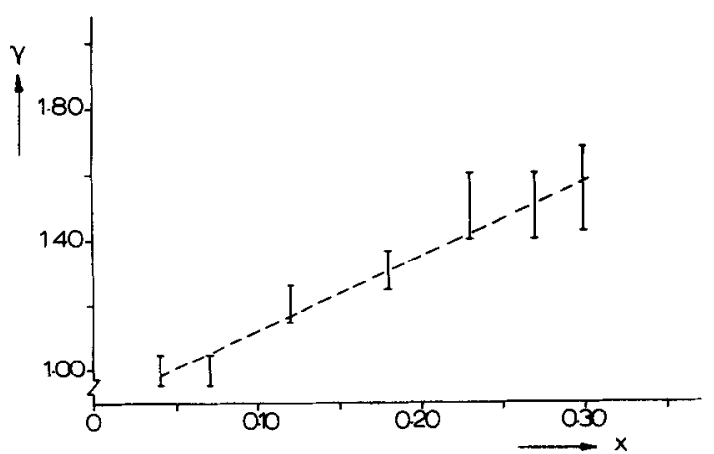

Fig. 4. The value of $y($ eqn 3$)$ as a function of the compositional parameter $x$ in $\mathrm{La}$ (III) substituted $\mathrm{PbTiO}_{3}$.

portant for grain size values $\bar{g}<3 \mu \mathrm{m}$ while the effects are more pronounced with smaller values of $\bar{g}$. Furthermore the change of the shape of the permittivity curves due to grain size effects in PLT cannot be described with eqn (3) which has a constant, temperature-independent $\gamma$-value. If grain size effects are present the $\gamma$-value in materials with small grain sizes changes with temperature over a small temperature region from $\gamma=\mathbf{2 . 0}$ near the FPT to $\gamma=1.0-1.3$ at higher temperatures. Macroscopic inhomogeneities result in phenomena of the same type.

The conclusion is that the change of $\gamma$ (and $C^{\prime}$ ) as expressed by eqn (3) is a property of monophasic, homogeneous, coarse grained materials which is due to compositional effects only.

\section{ALTERNATIVE DESCRIPTION AND CALCULATION MODEL}

An alternative description of the temperature dependency of the reciprocal dielectric constant can be achieved by the use of a power series of the temperature according to eqn (4)

$1 / \epsilon^{\prime}-1 / \epsilon_{\max }^{\prime}=C_{1}+C_{2}\left(T-T_{\max }\right)+C_{3}\left(T-T_{\max }\right)^{2}$.

Excellent agreement between experimental and calculated values (using eqn 4) are achieved at any temperature in the PE-phase with only two temperatureterms. This is shown in a representative way by Fig. 5 for PLT27. Higher order terms do not contribute to an improved accuracy and are negligibly small. The variance in the results achieved by a least square fit using eqn (4) is smaller than by using eqn (3).

In contrast to the use of eqn (4) a description where $\left(\epsilon^{\prime}-\epsilon_{\max }^{\prime}\right)$ is plotted as a function of the temperature leads to a poor agreement between experimental and calculated results especially near $T_{\max }$ where the calculated $\epsilon^{\prime}$-values diverge to infinity. Furthermore values of $C_{3}$ achieved from the $\left(\epsilon^{\prime}-\epsilon_{\max }^{\prime}\right)-T$ plots are always negative which makes a physical interpretation ver difficult (if at all possible).

The values of $C_{1}, C_{2}$ and $C_{3}$ are a function of the composition as presented in Table 2 . The value of $C_{2}$ decreases continuously with composition until $x=0.27$ and is about constant for $x \geqslant 0.27$. The values of $C_{3}$ increase with $x$ up to $x=0.12-0.18$ and decrease con-

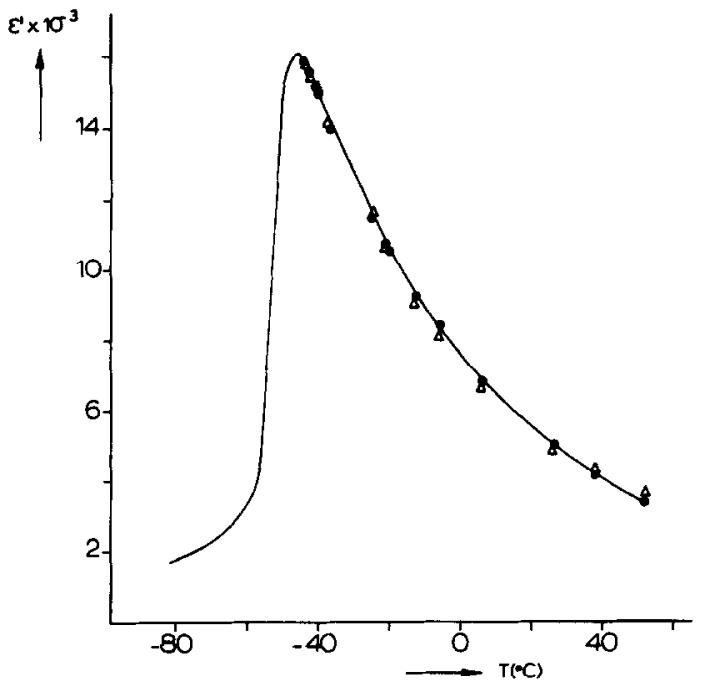

Fig. 5. The calculated and measured $\epsilon^{\prime}$ of PLT27 with $\alpha=1.48$ at $250 \mathrm{kHz}$ as a function of the temperature in the paraelectric phase $(-$, is measured; $\ldots \ldots$, is calculated with eqn (4); $\Delta \Delta \Delta \Delta \Delta$, is calculated with eqn (3)). For numerical values see Table 2.

tinuously until $x=0.30$. A grain size effect on the $C_{3}$ value for $x<0.07$ cannot be excluded (for $\bar{g}$-values see Table 1). Changes in $\alpha$-value (PbO content at a fixed $x$-value) play a minor role (compare samples 3 and 4 or 5 and 6) and will be ignored. At a low concentration of $x$ the linear term is dominant while at high $x$ values the reverse is true.

This result (eqn 4) can be derived assuming that the samples consist of a mixture of two types of regions, one in which eqn (1) and the other in which eqn (2) is obeyed. The existence of the latter region can be understood in terms of the presence of quasi-static fluctuations [20]. If $v$ is the volume fraction of material in regions of the first type, then, according to Bottcher [21], the Clausius-Mosotti relation can be written

$$
\frac{\epsilon^{\prime}-1}{\epsilon^{\prime}+2}=v \frac{\epsilon_{1}^{\prime}-1}{\epsilon_{1}^{\prime}+2}+(1-v) \frac{\epsilon_{2}^{\prime}-1}{\epsilon_{2}^{\prime}+2}
$$

Since the observed measurements indicate that all permittivities are much larger than one, the relationship can be expanded to terms of

$$
\frac{1}{\epsilon^{\prime}}=\frac{v}{\epsilon_{1}^{\prime}}+\frac{(1-v)}{\epsilon_{2}^{\prime}}
$$

When the appropriate terms from eqns (1) and (2) are inserted in eqn (6) and when the maximum value of $\epsilon^{\prime}$ at $T_{\max }$ of the region with diffuse character is labelled as $\epsilon_{2 \max }^{\prime}$ then we find

$1 / \epsilon^{\prime}=v \frac{\left(T-T_{0}\right)}{C_{w}}+(1-v)\left\{1 / \epsilon_{2 \max }^{\prime}+\frac{\left(T-T_{\max }\right)^{2}}{2 \epsilon_{2 \max }^{\prime} C^{2}}\right\}$.

The expression for the quadratic term (diffuse phase transition behaviour) has been derived by Wolters [4]. Kirillov and Isupov[8] and Smolensky [7] with different physical assumptions. After rearrangement it is clear that 
Table 2. The calculated values for $C_{1}, C_{2}$ and $C_{3}$ of eqn (4) and the calculated values for $v$ and $C$ of eqns (9) and (10) respectively for various samples of La(III) substituted $\mathrm{PbTiO}_{3}$

\begin{tabular}{ccccccc}
\hline $\begin{array}{c}\text { Sample } \\
\text { No. }\end{array}$ & \multicolumn{1}{c}{$C_{1} \times 10^{7}$} & $\begin{array}{c}C_{2} \times 10^{6} \\
\left(\boldsymbol{K}^{-1}\right) \\
\pm 0.20\end{array}$ & $\begin{array}{c}C_{3} \times 10^{8} \\
\left(\boldsymbol{K}^{-2}\right) \\
\pm 0.20\end{array}$ & $\begin{array}{c}f \\
(\mathbf{k H z})\end{array}$ & $\begin{array}{c}v \\
(-)\end{array}$ & $\begin{array}{c}C \\
(\mathbf{K})\end{array}$ \\
\hline 2 & 0.02 & 4.26 & 0.45 & 10 & 0.95 & 24 \\
3 & -0.44 & 2.95 & 2.19 & 10 & 0.66 & 28 \\
4 & -0.63 & 3.10 & 1.96 & 10 & 0.69 & 28 \\
5 & -0.09 & 1.93 & 3.20 & 10 & 0.43 & 30 \\
6 & -0.16 & 1.98 & 3.36 & 10 & 0.44 & 29 \\
7 & -0.06 & 0.66 & 1.84 & 10 & 0.15 & 48 \\
8 & 0.00 & 0.69 & 1.71 & 10 & 0.15 & 50 \\
8 & -0.11 & 0.79 & 1.51 & 250 & 0.18 & 52 \\
9 & 0.12 & 0.34 & 1.91 & 10 & 0.08 & 49 \\
9 & -0.04 & 0.48 & 1.53 & 250 & 0.11 & 54 \\
10 & -0.12 & 0.43 & 1.45 & 10 & 0.10 & 56 \\
10 & -0.48 & 1.05 & 1.07 & 250 & 0.23 & 60 \\
11 & -0.26 & 0.52 & 0.70 & 10 & 0.12 & 79 \\
11 & -1.48 & 0.74 & 0.61 & 250 & 0.10 & 86 \\
\hline
\end{tabular}

eqn (7) is equal to eqn (4) with

$$
\begin{aligned}
& C_{1}=\frac{(1-v)}{\epsilon_{2 \max }^{\prime}}-\frac{1}{\epsilon_{\max }^{\prime}}+\frac{v}{C_{W}}\left(T_{\max }-T_{0}\right) \\
& C_{2}=v / C_{W} \\
& C_{3}=(1-v) / 2 C^{2} \times \epsilon_{2 \max }^{\prime} .
\end{aligned}
$$

From eqn (9) the $v$-value can be calculated if the Curie-Weiss constant $C_{w}$ is known. Since the $C_{w}$-value does not change considerably in the PLT system it is taken to be independent of the concentration. The average value of $C_{w}$ is taken from eqn (4) for low $x$-values $(x=0.04$ and $x=0.07$ ) where the quadratic term can be ignored and therefore the value of $C_{w}=2.2 \times 10^{5}$ $\left({ }^{\circ} \mathrm{C}^{-1}\right)$ is taken for the whole composition region. The $v$-values calculated with eqn (9) have been collected in Table 2 and show a continuous decrease with increasing $\mathrm{La}(\mathrm{III})$ concentration from $v=0.95 \pm 0.005$ for $x=0.07$ to $v=0.12 \pm 0.08$ for $x=0.27$ and $x=0.30$.

Now the value of $C$ can be calculated with eqn (10) if $\epsilon_{2 \max }^{\prime}$ is known. The value of $\epsilon_{2 \max }^{\prime}$ is approximated by taking it as being equal to the average of $\epsilon_{\max }^{\prime}$ of the samples when $x=0.27$ and $x=0.30$ where diffuse behaviour dominates and therefore a value of $\epsilon_{2 \max }^{\prime}=$ 10,000 is used in the calculation. $\dagger$ The values of $C$ are shown in Table 2 and it can be seen that $C$ increases continuously with the La(III)-concentration. The increasing $C$-values reflect an increasing diffusivity of the regions with quadratic behaviour of the permittivity curve and of the same order of magnitude $(C=20-85)$ as reported for PLZT-materials [4] with a quadratic behaviour (diffuse phase transition). The changes in $v$ and

tA change in $\epsilon_{2 \max }$ of $20 \%$ gives a change in $\mathrm{C}$ of about $10 \%$. tThe degree of broadening $D$ can be calculated using the formula $D=3.25 \times k T_{c}^{2} /\left(Q \cdot v_{k}\right)$. Introducing values of the phase fluctuation volume $v_{k}=10^{-19}-10^{-17} \mathrm{~cm}^{3}$ (45-200 $\AA$ diameter) $[16,17]$ and using a measured value of the transition heat $Q \approx$ $11 \mathrm{cal} / \mathrm{mole}$ for PLT with $x=0.18$ calculated values of $D$ between $0.06 \mathrm{~K}$ and $6 \mathrm{~K}$, depending on $v_{k}$, are obtainable instead of the experimental value of more than $60 \mathrm{~K}$.
$C$ with $x$ remain qualitatively the same even with rather large changes in the values chosen for $C_{w}$ and $\epsilon_{2 \max }^{\prime}$.

\section{DISCUSSION OF THE CALCULATION MODEL}

The model described the material as consisting of a mixture of microregions where the effective volume fraction of the material with diffuse behaviour and the strength of the fluctuations within it (value of $C$ ) increases with increasing $\mathrm{La}(\mathrm{III})$ concentration $(x)$. With eqn (6) it is also possible to show why the permittivity curves are relatively sharp for $x \leq 0.23$ despite a high effective volume fraction of microregions with diffuse behaviour (with a characteristic shape of the permittivity curve as presented in Fig. 3). Introduction of the temperature dependence of $\epsilon_{1}^{\prime}$ and $\epsilon_{2}^{\prime}$ in eqn (6) leads to a sum curve $\epsilon_{3}^{\prime}$ as shown in Fig. 6. The temperature dependence of $\epsilon_{3}^{\prime}$ is mainly determined by the term with the lowest permittivity $\left(\epsilon_{1}^{\prime}\right)$ especially in the ferroelectric phase. If the value of $v$ approaches zero then a sum curve with a more diffuse character as shown in Fig. 3 appears.

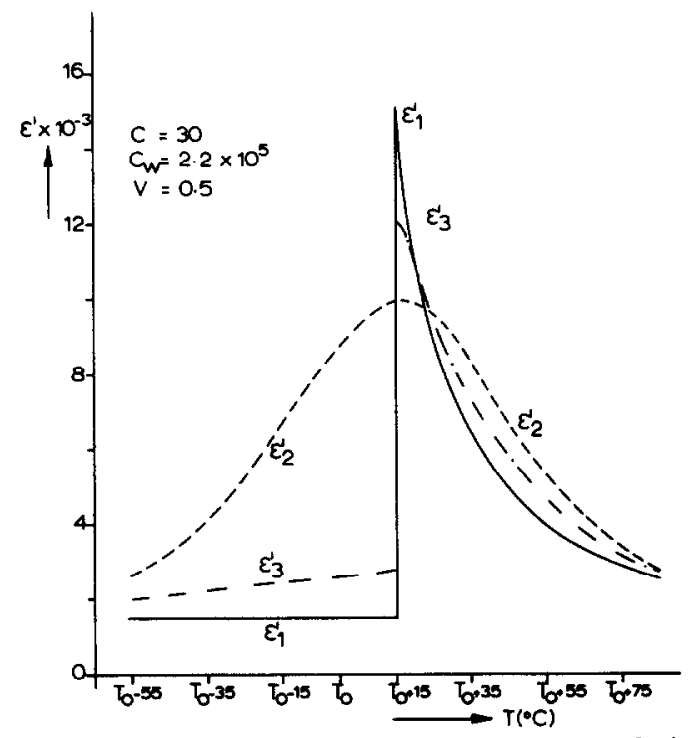

Fig. 6. The graphical representation of eqn (6). (1, "CurieWeiss" curve; 2 , "quadratic" curve; 3 , "sum" curve.

The physical basis of the above mentioned model is the occurrence of fluctuations in the structure. A description of the possible fluctuations (static or dynamic, polarization and/or composition) has been given by Wolters [4]. They can all give rise to a quadratic term in the permittivity curve. No definite conclusion can be drawn with respect to the exact type of fluctuation.

Arguments against the occurrence of dynamic fluctuations in a large temperature region are (1) the absence of a rather strong frequency-dependency of the permittivity and (2) a calculated degree of broadening, using the formula of Rolov and Fritsberg[6, 16,17], which is much smaller than the experimental values obtained from the permittivity curves. $\neq$

Indication for the possible existence of rather static compositional fluctuations is the tendency for ordering in $\mathrm{La}$ (III) substituted $\mathrm{PbTiO}_{3}$ with increasing $\mathrm{La}(\mathrm{III})$ 
fluctuations in space.

Recently Meitzler[19] discussed the possible occurrence of crystallographic shear processes in these types of materials which are based on the occurrence of ordered planes of vacancies in certain areas of the structure.

\section{REFERENCES}

1. Blinc R. and Zeks B., Soft Modes in Ferroelectrics and Antiferroelectrics, Chap. I. North Holland, Amsterdam, Oxford (1974).

2. Burfoot J. C., Ferroelectrics, Chap. I. D. van Nostrand, London (1967).

3. Fatuzzo E. and Merz W. J., Ferroelectricity, Chap. 2, North Holland, Amsterdam (1967).

4(a) Wolters M. and Burggraaf A. J., Mat. Res. Bull. 10, 417

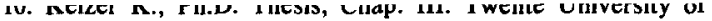
Technology, Enschede, The Netherlands (1976).

11. Kruidhof H. and de Vries K. J., An. Chim. Acta, to be published.

12. Mendelson M. I., J. Am. Ceram. Soc. 52, 443 (1969).

13. Keizer K. and Burggraaf A. J., Ferroelectrics 14, 671 (1976).

14. Keizer K. and Burggraaf A. J., Phys. Status Solidi (a) 26, 561 (1974).

15. Burggraaf A. J. and Keizer K., Mat. Res. Bull. 10, 521 (1975).

16. Rolov B. N., Bull. Acad. Sci. USSR, Phys. Ser. 33, 205 (1969).

17. Rolov B. N., J. Phys. C2. Suppl. 33, 257 (1972)

18. Bouwma J., de Vries K. J. and Burggraaf A. J., Phys. Status Solidi (a) 35, 281 (1976).

19. Meitzler A. H., Ferroelectrics 11, 503 (1976).

20. Cook M. E., J. Appl. Cryst. 8, 132 (1975).

21. Bötcher C. T. F., Theory of Electric Polarization, Vol. I. Chap. 5. Elsevier, Amsterdam (1973). 